Editorial

\title{
Comunicación para la salud laboral
}

\section{Communication for Occupational Health}

\author{
Javier Sanz-Valero' \\ Servicios. Madrid, España. \\ Recibido: 17-06-2019 \\ Aceptado: 20-06-2019

\section{Correspondencia} \\ Javier Sanz-Valero \\ Instituto de Salud Carlos IIII \\ Escuela Nacional de Medicina del Trabajo, Área de Divulgación e Investigación y Servicios. \\ Madrid, España \\ Correo electrónico: fj.sanz@isciii.es
}

1. Instituto de Salud Carlos IIII. Escuela Nacional de Medicina del Trabajo, Área de Divulgación e Investigación y

La comunicación es esencial a la naturaleza y práctica de la ciencia. Los científicos no sólo comunican los resultados a sus colegas, sino que también se apoyan en el conocimiento de trabajos previos para formular propuestas y metodologías de investigación. El intercambio de opiniones y datos con los pares es parte esencial de la fase experimental. Por lo tanto, la comunicación está presente en todas las etapas de la investigación. Ahora bien, una de las características propias del conocimiento científico es que debe ser público y consensuado. Es decir, la ciencia debe ser comunicable; si un cuerpo de conocimiento no lo es, entonces por definición no es científico. La comunicabilidad es a su vez una condición necesaria para la verificación de los datos empíricos y de las hipótesis científicas ${ }^{1}$.

La comunicación en salud se podría definir como el arte y las técnicas para informar, influir y motivar al público sobre temas de salud relevantes desde la perspectiva individual, comunitaria e institucional y englobaría la prevención de enfermedades, las políticas de salud y la mejora de la calidad de vida de las personas y las comunidades.

La Organización Mundial de la Salud (OMS) en su documento "Marco estratégico de la OMS para las comunicaciones eficaces $»^{2}$, reconoce que la comunicación efectiva, integrada y coordinada es esencial para llevar a cabo el objetivo de la OMS de construir un futuro mejor y más saludable para las personas en todo el mundo. El propósito de este documento es describir un enfoque estratégico para comunicar, de manera efectiva, la información, el asesoramiento para una amplia gama de problemas de salud: desde enfermedades crónicas hasta la aparición de riesgos emergentes.

Los seis atributos, o principios básicos, que la OMS estableció para la adecuada comunicación fueron: accesible, factible, creíble y fiable, pertinente, a tiempo y fácil de entender. Y, se representan, en relación a los posibles interlocutores, en la figura 1.

La Organización de las Naciones Unidas para la Educación, la Ciencia y la Cultura (UNESCO) reconoció, en la «Declaración de la Ciencia y el Uso del Conocimiento Científico», adoptada en la Conferencia Mundial

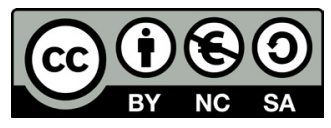

https://creativecommons.org/licenses/by-nc-sa/4.0/ 
de la Ciencia de $1999^{3}$, la importancia de que los científicos se involucraran activamente en la comunicación con la sociedad.

Figura 1. Esquema propuesto por la Organización Mundial de la salud para lograr una adecuada comunicación para la salud.

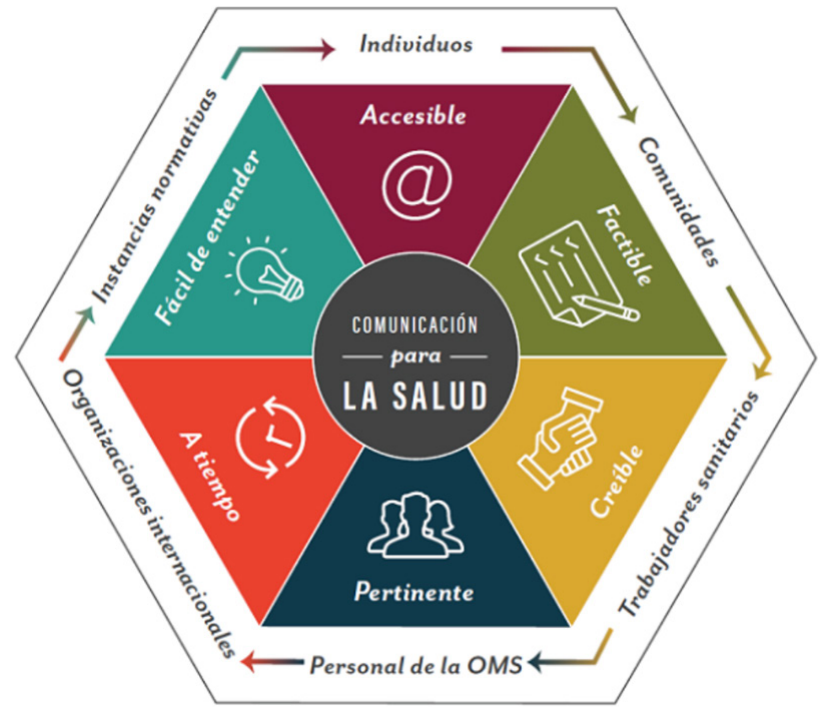

En el año 2010, por primera vez, la comunicación de salud tuvo un capítulo en los objetivos de Healthy People 2010 de los Estados Unidos de América ${ }^{4}$. En estos objetivos se consideró que la comunicación para la salud tenía especial relevancia para prácticamente todos los aspectos del bienestar de las personas, incluida la prevención de enfermedades, la promoción de la salud y la calidad de vida. Con ello, los profesionales de la comunicación sanitaria reconocían la importancia de la prevención y, con ella, la necesidad de comprender el comportamiento humano ante el riesgo para su salud5.

Al fin y al cabo, la comunicación es el núcleo de lo que somos como seres humanos, es nuestra manera de intercambiar información, pero también lleva aparejada nuestra capacidad simbólica ${ }^{6}$. Estas dos funciones reflejan lo que Carey identificaba como la transmisión y puntos de vista rituales de la comunicación, y señalaba que la comunicación desempeña un papel instrumental (ayuda a tener conocimiento) y, además, cumple una función ritual, (refleja a los seres humanos como miembros de una comunidad social) ${ }^{7}$. Por lo tanto, la comunicación puede ser definida como el intercambio simbólico de sentido común y todos los actos comunicativos tener tanto una transmisión de información como un componente ritual.

Divulgar el conocimiento está intrínsecamente ligado a nuestra capacidad para dar respuesta a la innata curiosidad con que perseguimos intentar entender la complejidad del mundo en que vivimos. En consecuencia, la diseminación de la cultura científica se ha convertido en un indispensable catalizador que debe llevar a una cohesionada sociedad del conocimiento. Pero, el mundo científico y los medios de comunicación emprendieron, o deberían haberlo realizado, la difícil adaptación a la sociedad de la información en red. Hoy, hay que ir mucho más allá de las formas tradicionales de compartir y sociabilizar el conocimiento; por ello, comunicar en red ya se ha convertido en una estrategia esencial, complementaria e indisociable . $^{8}$

En esta etapa de transición hacia el paradigma informacional, Internet ha llegado para quedarse y ha revolucionado la forma en que la población recibe la información. En esta tarea informativa, pero también con un fuerte componente educativo, las herramientas 2.0 (blogs, wikis y redes sociales) se han convertido en un nuevo medio que permite a más personas llegar a una "buena" información generada por un sistema cooperativo. Habilita a los usuarios para ser participantes activos coproduciendo contenidos, aumentando la conectividad y la colaboración en la producción de nuevo conocimiento?

\section{LA COMUNICACIÓN EN LA SALUD LABORAL}

La comunicación efectiva sobre los riesgos para la salud es una herramienta importante que puede prevenir o modificar las acciones inapropiadas que a menudo motivan los problemas de la salud laboral. La percepción pública de la magnitud o importancia del riesgo, a menudo, es diferente a la evidencia científica. 
Por tanto, el objetivo de la comunicación en la salud laboral debe ser algo más que transmitir datos científicos. Se trata de conseguir que la población comprenda plenamente el riesgo y que pueda tomar decisiones informadas en condiciones de peligro o incertidumbre. La forma en que se percibe un peligro, sus valores y sentimientos hacia los problemas de salud laboral, son consideraciones tan importantes, para la comunicación del riesgo, como lo son los datos científicos numéricos o fácticos.

Parra $^{10}$, ya en 2003, destacaba la importancia de la comunicación en el trabajo: «La comunicación también es un aspecto esencial de la convivencia humana y, en ese sentido, el espacio de trabajo es un lugar de convivencia entre personas, donde se crean redes de apoyo, amistades, se forman y fortalecen familias, entre otras importantes derivaciones de este carácter social del trabajo. Por lo mismo, favorecer la comunicación en el trabajo mejora la satisfacción y el conocimiento de las personas.

Lo habitual es que el trabajador reciba una serie de instrucciones para realizar su trabajo. En un caso óptimo dicha información además incluye advertencias sobre precauciones que debe tomar para proteger su seguridad y la de las demás personas. Asimismo, el trabajador cuenta con alguna forma de transmitir información relevante hacia los superiores jerárquicos. La forma en que se transmite la información, su claridad y la capacidad de las personas para comprenderla son importantes herramientas para una mejor productividad y una adecuada prevención de accidentes y enfermedades».

Los problemas para la comunicación en el ámbito de la salud laboral de deben a las complicaciones tales como la multidimensionalidad del peligro, la confianza en las fuentes de información, la revolución tecnológica, la dependencia de los medios de información de salud y el deseo público de información verídica. Si, los profesionales de la salud, quieren abordar de manera efectiva los problemas de salud laboral tanto reales como percibidos, deben ser conscientes de los principales avances que se han realizado en el uso de la comunicación de riesgos en los últimos años ${ }^{11}$. De lo contrario, la persona receptora pensará que esta información es, desde su principio, obsoleta o, lo que es peor, poco fiable.

Así la salud laboral debe aprovechar más el desarrollo de las tecnologías de la información y las comunicaciones (TIC), las que han modificado los esquemas del mundo, por su utilidad en la gestión y la promoción de productos y servicios ${ }^{12}$.

Las TIC representan una superación de diversas barreras relacionadas con el tiempo y el espacio; se redefine así el contexto físico y social del trabajo en relación con el grupo de trabajo, la supervisión, la propia vida y el entorno familiar ${ }^{13}$.

En nuestros días, es posible actuar en la Red desde espacios diferentes en horarios diferentes. Se trastoca el hasta hace poco indivisible trinomio acción/espacio/tiempo. En el mundo del conocimiento se puede acceder a un montón de documentos multimedia, de mayor o menor calidad, a través de múltiples caminos. Sin embargo, conocer su verdadero valor y aptitud es un privilegio que la sociedad debe tener. ¡Este es su reto! ${ }^{14}$

Conviene recordar que, en la 60. ${ }^{a}$ Asamblea Mundial de la Salud, en el apartado WHA60.26 Salud de los trabajadores: plan de acción mundial ${ }^{15}$, puede leerse: «... Es preciso desarrollar, con la participación de todas las partes interesadas, estrategias e instrumentos para mejorar la comunicación y promover la toma de conciencia acerca de la salud de los trabajadores. Esas estrategias e instrumentos deben estar orientados a los trabajadores, los empleadores y sus organizaciones; los formuladores de políticas; el público en general, y los medios de comunicación...».

Así pues, la promoción de la comunicación es el mejor sustento de las prioridades científico-técnicas en salud laboral y para fomentar el diálogo entre los involucrados en el desarrollo de la misma ${ }^{12}$.

Vega Ramírez et al. ${ }^{16}$, señalaban que una buena comunicación, junto con el apoyo social, se presentan como la correlación más fuerte en un ambiente de trabajo saludable, apoyando un modelo integral que intervenga en la promoción de la salud dirigida a las condiciones de trabajo, las relaciones laborales y su organización.

En conclusión, la comunicación en salud laboral tiene aún trabajo por hacer y aunque va avanzando y ganando reconocimiento, es en parte debido a su énfasis en la combinación de la teoría y la práctica en la comprensión de los procesos de comunicación y el cambio de la conducta humana. Este enfoque es pertinente en un momento en que muchas de las amenazas para la salud pública mundial (a través de las enfermedades y calamidades ambientales) tienen su origen en el comportamiento humano. 
Para acabar una apreciación, tomada de la publicación de Rodrigo de Larrucea ${ }^{4}$, en un momento en que todos los expertos hablan de comunicación, de datos de salud, de aplicaciones informáticas, de inteligencia artificial, etc..., es importante reconocer que al profesional de la salud laboral se le forma para prevenir, auditar, diagnosticar o tratar, pero, no se le entrena para comunicar ¿sería necesario introducir en la enseñanza reglada de estos profesionales formación en comunicación, aunque suene paradójico?

\section{BIBLIOGRAFÍA}

1. Sanz-Lorente M, Guardiola-Wanden-Berghe R. Comunicar la ciencia. Hosp Domic. 2019;3(2):173-83. DOI: 10.22585/hospdomic. v3i2.57

2. Department of Communications. WHO strategic communications framework [monografía en Internet]. Geneva, Switzerland: World Health Organization; 2017 [citado 4 de abril de 2020]. Disponible en: https://bit.ly/3bVBZDM

3. Organización de las Naciones Unidas para la Educación, la Ciencia y la Cultura (UNESCO). Declaración de la Ciencia y el Uso del Conocimiento Científico, adoptada en la Conferencia Mundial de la Ciencia de 1999 [monografía en Internet]. Budapest, Hungría: UNESCO; 1999 [citado 6 de abril de 2020]. Disponible en: https://bit.ly/2JFKiHC

4. National Center for Health Statistics. Healthy People 2010 [monografía en Internet]. Atlanta, USA: Centers for Disease Control and Prevention; 2015 [citado 5 de abril de 2020]. Disponible en: https://bit.ly/3aRbFKM

5. Rimal RN, Lapinski MK. Why health communication is important in public health. Bull World Health Organ. 2009;87(4):247.

6. Rodrigo de Larrucea C. Comunicación en salud pública. En: Rodrigo de Larrucea C, editor. Derecho y salud no van siempre de la mano [monografía en Internet]. Barcelona, España: Digestum Legal; 2016 [citado 5 de abril de 2020]. Disponible en: https:// bit.ly/2V9Pf0R

7. Carey JW. Communication as culture: Essays on media and Society. New York, USA: Routledge; 1992.

8. de Semir V, Revuelta G. Cuadernos de la Fundación Dr. Antonio Esteve No 20: La importancia de la comunicación en el entorno científico [monografía en Internet]. Barcelona, España: Fundación Dr. Antonio Esteve; 2010 [citado 5 de abril de 2020]. Disponible en: https://bit.ly/39Q1RiO

9. de Semir V, Revuelta G. Cuadernos de la Fundación Dr. Antonio Esteve No 25: Información médica y educación sanitaria en la revolución de las TIC [monografía en Internet]. Barcelona, España: Fundación Dr. Antonio Esteve; 2012 [citado 6 de abril de 2020]. Disponible en: https://bit.ly/2V3s5sM

10. Parra M. Conceptos básicos en salud laboral. Santiago, Chile: Organización Internacional del Trabajo; 2003.

11. Nicholson PJ. Communicating occupational and environmental issues. Occup Med (Lond). 2000;50(4):226-30. DOI: 10.1093/ occmed/50.4.226

12. del Castillo Martín NP. Perfeccionamiento de los procesos de comunicación en el subsistema de salud ocupacional. Rev Cub Salud Trab. 2014;15(3):48-57.

13. Gómez IC. Salud laboral: una revisión a la luz de las nuevas condiciones del trabajo. Univ Psychol. 2007;6(6):105-13.

14. Sanz-Valero J, Castiel LD, Wanden-Berghe C. Las aventuras de Alicia en el maravilloso mundo del conocimiento: el camino hacia la actual alfabetización. Hist Cienc Saude-Manguinhos. 2010;17(1):153-64. DOI: 10.1590/S0104-59702010000100010

15. Organización Mundial de la Salud (OMS). 60ª Asamblea Mundial de la Salud - WHA60.26 Salud de los trabajadores: plan de acción mundial. Geneva, Switzerland: OMS; 2007.

16. Vega Ramírez FA, Catalán Matamoros D, López Liria R. El rol de las campañas de comunicación en la promoción de la salud y la prevención de lesiones en salud laboral. Rev Esp Com Sal. 2010;1(2):137-54. 